\title{
Toward Tetherless Insufflation of the GI Tract
}

\author{
Jenna L. Toennies, Member, IEEE, Gastone Ciuti Member, IEEE, Byron F. Smith, Member, IEEE, \\ Arianna Menciassi, Member, IEEE, Pietro Valdastri Member, IEEE, and Robert J. Webster III, Member, IEEE
}

\begin{abstract}
Toward increasing the diagnostic ability of wireless capsule endoscopy, we propose a method to wirelessly insufflate the Gastrointestinal Tract. By increasing the viewable surface area, it appears likely that capsule-based insufflation may reduce the number of false negative diagnoses made by endoscopic capsules. Our approach to wireless insufflation is to utilize controlled phase transition of a small volume of fluid stored onboard the capsule to a large volume of gas that is then emitted into the intestine. We begin by describing experiments designed to evaluate the amount of gas a capsule must produce to have a beneficial impact on visualization in the colon. We then describe experiments evaluating how much gas can be generated from a given volume of fluid, using Hydrogen Peroxide as our working fluid. We also evaluate thermal effects of the Hydrogen Peroxide reaction. The cumulative result of these experiments is an illustration of the feasibility of carrying a sufficient volume of fluid onboard a wireless capsule to generate a beneficial enhancement in visualization of the interior of the Gastrointestinal Tract, and specifically the colon.
\end{abstract}

\section{INTRODUCTION}

Passive commercial capsules such as the PillCam (Given Imaging, Inc.) and competing devices are rapidly becoming the gold standard for diagnosis of many diseases including Crohn's Disease, Celiac Disease, Obscure Gastrointestinal Bleeding, and cancer, all of which occur in the small intestine [1]. Because of their successes in the small intestine, capsules are being developed for other areas of the Gastrointestinal (GI) Tract, such as the esophagus, the stomach, and the large intestine [2]. Researchers have also begun exploring ways to robotically enhance the diagnostic and interventional capabilities of these capsules. Although the development of miniature sensors, actuators, and mechanisms is challenging, many innovative designs have been created [2]-[4].

One common challenge with which all endoscopic capsules must contend is the distention of tissue away from the device, and particularly away from the face of the onboard camera (see Figure 1). This is especially important in the large intestine, where the intestinal lumen is much larger than the capsule diameter. Traditional endoscopes achieve distention by inflating the intestine with air. Such insufflation provides a much clearer view of the wall of the intestine, as can be seen in Figure 1. Our aim in this work is to bring the advantages of insufflation to bear on wireless capsule endoscopes, for enhancing visualization. To achieve this, we propose chemofluidic phase transition as a means of generating

J.L. Toennies, B.F. Smith, and R.J. Webster III are with Vanderbilt University, Nashville, TN, 37235 USA (e-mail: \{jenna.l.toennies, byron.smith, robert.webster\}@ vanderbilt.edu).

G. Ciuti, A. Menciassi, and P. Valdastri, are with Scuola Superiore Sant'Anna, Pisa, Italy (e-mail: \{g.ciuti, arianna.menciassi@sssup.it, pietro.valdastri\}@sssup.it).
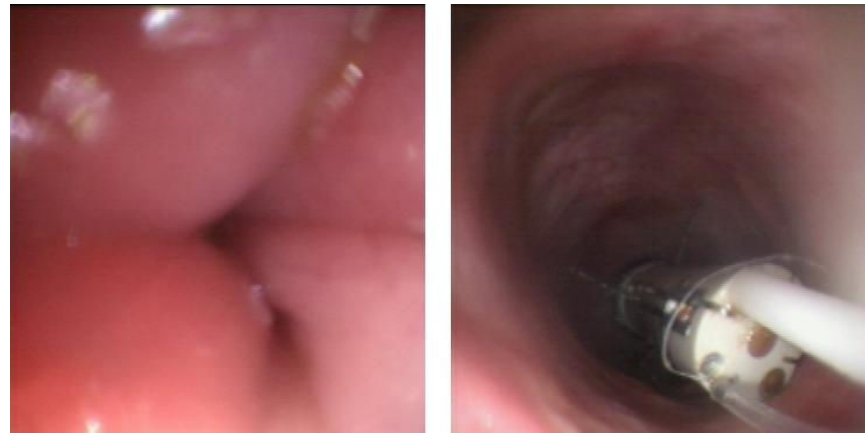

Fig. 1. (Left) An image from a colonoscope of the colon prior to insufflation. (Right) An image after insufflation. Note that in the right image more of the intestinal surface can be visualized. Also pictured is a prototype of a capsule robot with legs [5].

large volumes of gas from small volumes of fluid carried onboard the capsule.

To evaluate the feasibility of tetherless insufflation, in this paper we explore (1) how much gas must be generated to tangibly enhance visualization in the colon, and (2) whether it is feasible to carry a sufficient volume of liquid Hydrogen Peroxide $\left(\mathrm{H}_{2} \mathrm{O}_{2}\right)$ onboard a swallowable capsule to enhance visualization.

\section{COLON INFLATION EXPERIMENT}

To assess the volume of gas that must be generated to measurably enhance visualization in the colon, we performed the following experiment, using the experimental setup shown in Figure 2. Nine colored markers serving as identifiable fiducials were evenly spaced and attached around the internal intestinal surface of porcine large intestine (see Figure 3(e)), with dimensions representative of an average adult colon

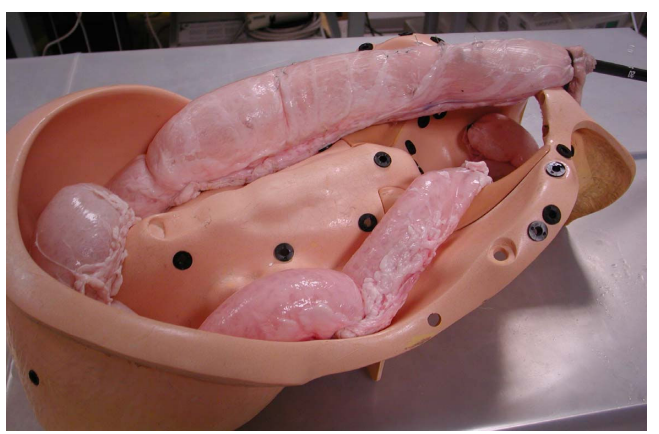

Fig. 2. The experimental setup for exploring how much gas volume is needed to inflate the intestine. Ex vivo porcine intestine was arranged in a phantom model to simulate the shape of the human colon. An endoscope was then used to inject known volumes of air, while the surface area visible on the interior wall was measured in the endoscope images (see Figure 3). 

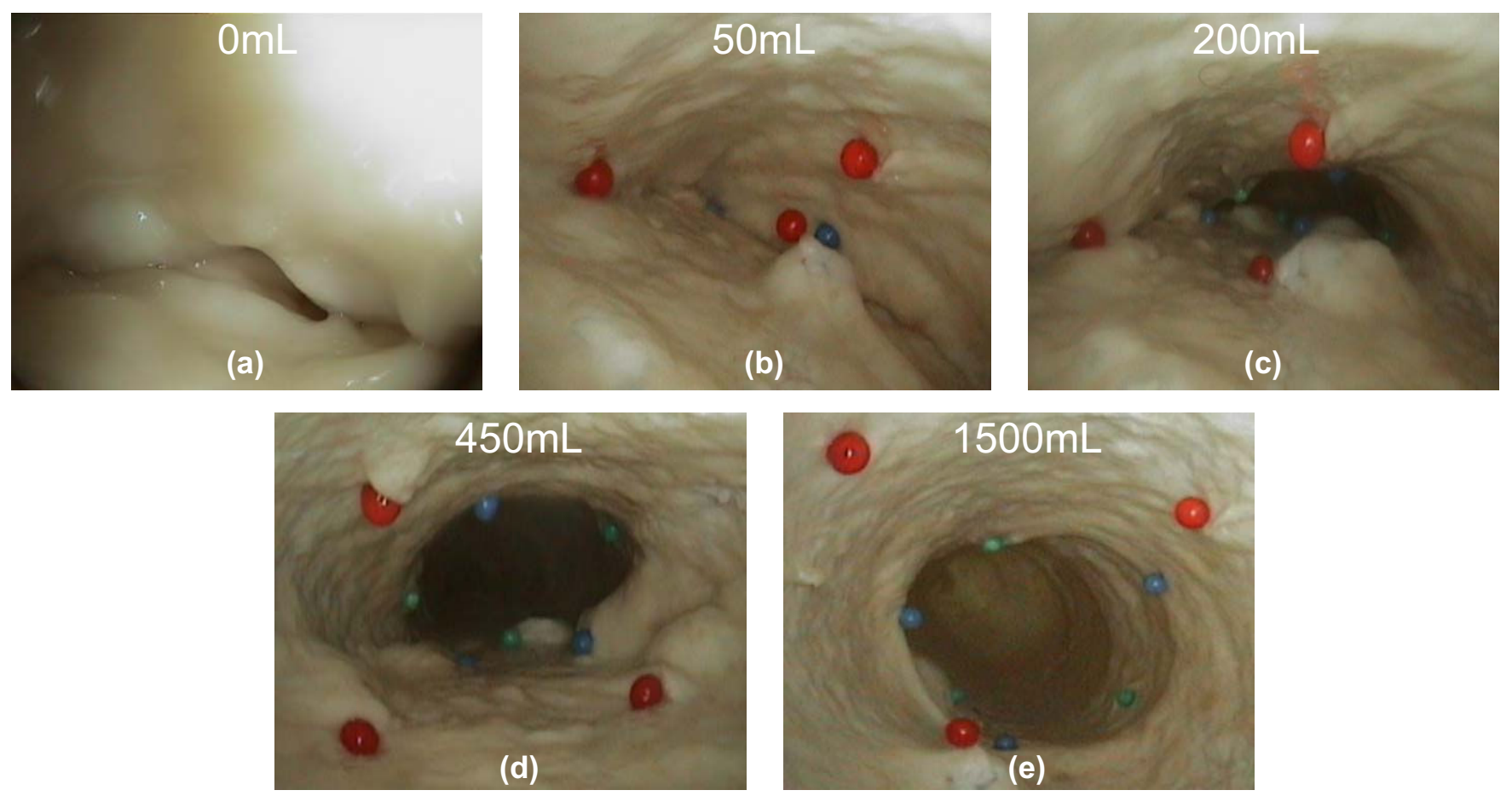

Fig. 3. The results of the insufflation experiment. (a) The intestine in its deflated state with no markers visible. (b) With just $50 \mathrm{~mL}$ of insufflation, 4 of the 9 markers became visible. (c) At $200 \mathrm{~mL}$, all 9 markers first come into the field of view. (d) The threshold above which all 9 markers were consistently visible was $450 \mathrm{~mL}$. (e) The intestine in its fully inflated state at $1500 \mathrm{~mL}$ of insufflation.

TABLE I

THE NUMBER OF MARKERS VISIBLE (OUT OF 9 TOTAL) FOR EACH INFLATION VOLUME. ABOVE 450ML ALL 9 MARKERS WERE VISIBLE.

\begin{tabular}{|c|c|}
\hline Air Volume $(\mathrm{mL})$ & Markers Visible \\
\hline 0 & 0 \\
\hline 50 & 4 \\
\hline 100 & 5 \\
\hline 150 & 8 \\
\hline 200 & 9 \\
\hline 250 & 8 \\
\hline 300 & 7 \\
\hline 350 & 9 \\
\hline 400 & 7 \\
\hline 450 & 9 \\
\hline 1500 & 9 \\
\hline
\end{tabular}

$(150 \mathrm{~cm}$ long, $6 \mathrm{~cm}$ in diameter) [6]. A flexible endoscope (13803PKS, STORZ) was inserted in one end of the intestine, and then both ends were tightly sealed. The intestine was incrementally inflated through a channel in the endoscope using a controlled air compressor. An in-line flow sensor (AWM3300V, Honeywell) was used to record volume, and images were captured using the endoscope camera at each volume increment. The number of markers visible at each increment is shown in Table I.

\section{Gas Generation from Hydrogen Peroxide}

Using the air volumes found in the inflation experiment, we now determine the necessary fluid volume to produce each. Hydrogen Peroxide is a promising working fluid because it can produce a large volume of gas from a small initial fluid volume (see Figure 4). To generate gas from $\mathrm{H}_{2} \mathrm{O}_{2}$, the capsule must simply pass liquid $\mathrm{H}_{2} \mathrm{O}_{2}$ through a catalyst (e.g. a silver or platinum screen), which catalyzes the conversion to oxygen gas and water.

To calculate the necessary volume of fluid for a given volume of gas, one might initially think that a simple computation using the chemical decomposition equation and the Ideal Gas Law would suffice. However, there are many factors that are not accounted for in such an ideal calculation. Temperature and concentration of $\mathrm{H}_{2} \mathrm{O}_{2}$ (which affects both the temperature of the reaction and the amount of gas generated) are both variable during the reaction. Furthermore, some water is converted to vapor during the reaction, which depends on temperature and concentration. Thus, calculations that do not include any water evaporation underestimate the gas generation by a factor of almost 2 at high concentrations. For this reason, we conducted experiments to determine the amount of gas generated by reacting small volumes of $\mathrm{H}_{2} \mathrm{O}_{2}$ at various concentrations, as described in the following section.

\section{Peroxide Reaction Experiment}

As discussed above, a tradeoff exists between the concentration of $\mathrm{H}_{2} \mathrm{O}_{2}$ used and the temperature of the reaction. A higher concentration produces more gas, but also generates more heat, which must be safely dissipated without burning the intestine. In order to investigate the effects of concentration on the gas volume and temperature of the reaction, we experimentally reacted known fluid volumes and measured output gas for $30 \%, 50 \%$, and $70 \%$ solutions $\mathrm{H}_{2} \mathrm{O}_{2}$.

The experimental setup, shown in Figure 5, involved a mixing flask, a holding flask, and a discharge cylinder. The 


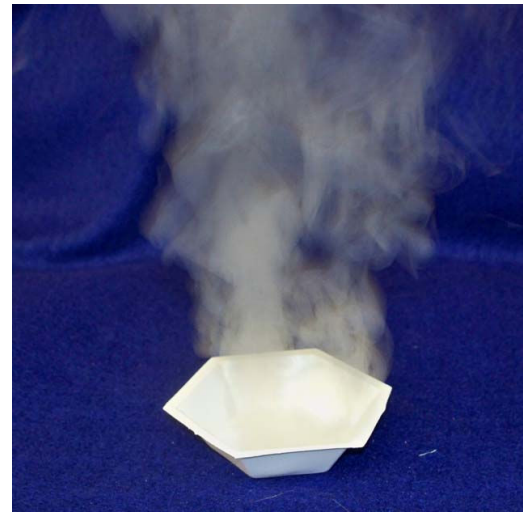

Fig. 4. A large amount of Oxygen was produced when a small amount of liquid Hydrogen Peroxide was catalyzed by a silver screen.

flasks were connected together with rubber tubing and were sealed with rubber stoppers. A thermocouple was used to measure the maximum temperature of the reaction (see Figure 7). The catalyst used was a fine silver screen mesh, and it was cut into $11 \mathrm{~mm}$ circles to replicate the maximum sized screen that could fit within a swallowable capsule. An initial amount of $\mathrm{H}_{2} \mathrm{O}_{2}$ was placed in the mixing flask. The catalyst was dropped into the flask, and the flask was quickly sealed. The gas produced from this reaction displaced an approximately equal volume of water in the holding flask, which was ejected through a plastic tube and a check valve, into a graduated cylinder where the volume was measured.

This test was performed with initial volumes ranging from $0.5-1.25 \mathrm{~mL}$ (in $0.25 \mathrm{~mL}$ increments) for three concentrations of $\mathrm{H}_{2} \mathrm{O}_{2}(30 \%, 50 \%$, and $70 \%)$. To ensure repeatability, three trials at each increment were performed. One catalyst screen was used for each increment (i.e. one screen was used 3 times at $0.5 \mathrm{~mL}$, and a new screen was used 3 times at $0.75 \mathrm{~mL}$, and

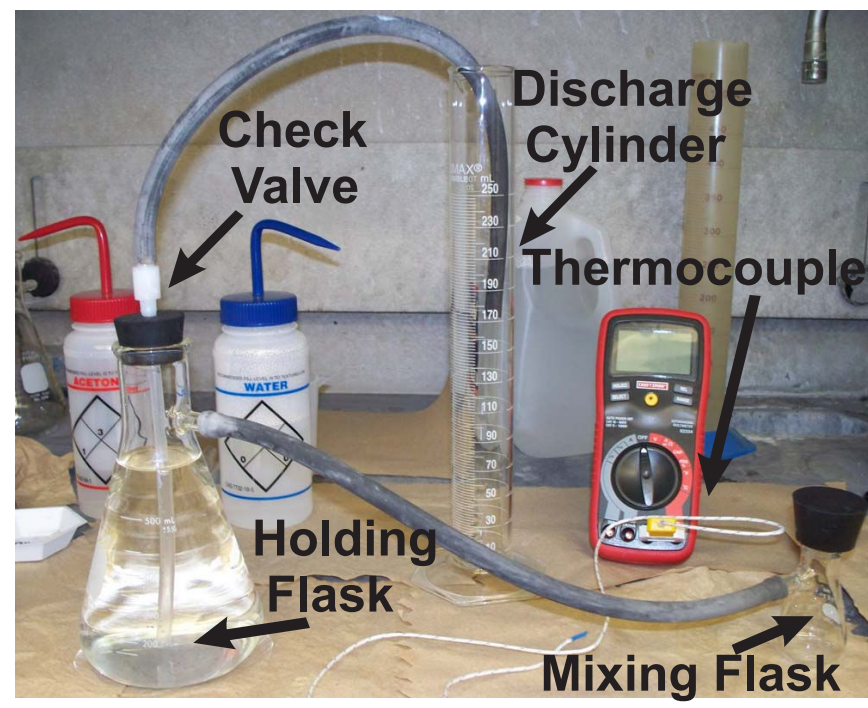

Fig. 5. Experimental setup for reacting known volumes of $\mathrm{H}_{2} \mathrm{O}_{2}$ to measure gas production. A silver screen catalyst was dropped into the mixing flask which held a small initial volume of $\mathrm{H}_{2} \mathrm{O}_{2}$. The gas generated displaced water in the holding flask, which was measured in a graduated cylinder.

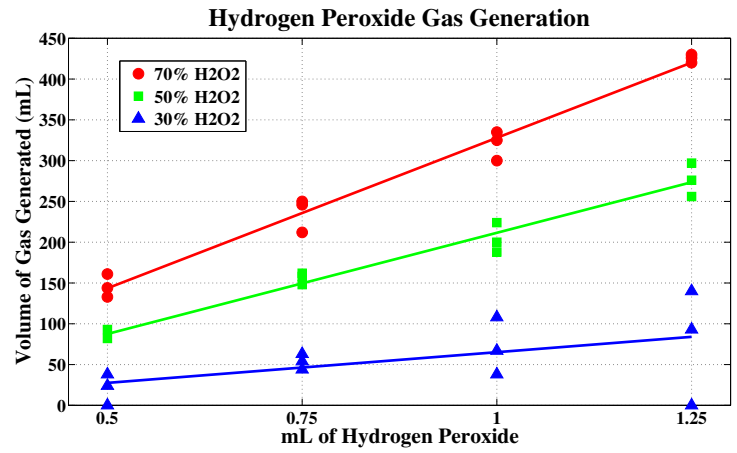

Fig. 6. The volume of gas produced $(\mathrm{mL})$ for initial volumes of liquid Hydrogen Peroxide at varying concentrations.

so forth). The output volume of gas (i.e. the water volume in the graduated cylinder) was recorded for each experiment, as shown in Figure 6. A linear fit to this data was used to compute the initial fluid volumes of $\mathrm{H}_{2} \mathrm{O}_{2}$ a capsule would need to carry to generate the desired inflation levels from the inflation experiment described in Section II. Results are given in Table II.

\section{DISCUSSION}

As can be seen in Figure 3, at just $50 \mathrm{~mL}$ of inflation, visualization was significantly improved with 4 of the 9 markers becoming visible. At this point the required initial fluid volume was less than $0.710 \mathrm{~mL}$ for all concentrations of $\mathrm{H}_{2} \mathrm{O}_{2}$. For comparison, note that commercial capsules such as the PillCamSB (Given Imaging, Inc.) have total volumes of approximately $2.47 \mathrm{~mL}$. An initial conceptual prototype insufflation capsule, constructed from off-the-shelf components, contained a fluid reservoir of $0.615 \mathrm{~mL}$ [7], and it is likely that this fluid reservoir size can be increased by minor design changes (i.e. changing component locations and reducing wall thicknesses). Thus, these results indicate that it is feasible for capsules to carry a sufficient volume of fluid to achieve visualization benefits, since Figure 3 indicates that they start with as little as $50 \mathrm{~mL}$ of insufflation delivered. With $200 \mathrm{~mL}$ of insufflation, the intestine is almost fully inflated, and all 9 markers first became visible (see Figure 3(c)), yet the required fluid volumes remain relatively small. Even the upper threshold of $450 \mathrm{~mL}$, above which all markers were always

TABLE II

ONBOARD CAPSULE FLUID VOLUMES REQUIRED TO PRODUCE CORRESPONDING GAS VOLUMES FROM THE INSUFFLATION EXPERIMENT. THE TYPICAL VOLUME OF A COMMERCIAL CAMERA PILL IS $2.47 \mathrm{ML}$.

\begin{tabular}{|c|c|c|c|}
\hline Air $(\mathrm{mL})$ & $\mathrm{H}_{2} \mathrm{O}_{2} 70 \%(\mathrm{~mL})$ & $\mathrm{H}_{2} \mathrm{O}_{2} 50 \%(\mathrm{~mL})$ & $\mathrm{H}_{2} \mathrm{O}_{2} 30 \%(\mathrm{~mL})$ \\
\hline 50 & 0.2571 & 0.3488 & 0.7103 \\
\hline 100 & 0.3910 & 0.5504 & 1.167 \\
\hline 150 & 0.5248 & 0.7520 & 1.6233 \\
\hline 200 & 0.6586 & 0.9536 & 2.0798 \\
\hline 250 & 0.7925 & 1.1552 & 2.5363 \\
\hline 300 & 0.9263 & 1.3569 & 2.9928 \\
\hline 350 & 1.0601 & 1.5585 & 3.4492 \\
\hline 400 & 1.1940 & 1.7601 & 3.9058 \\
\hline 450 & 1.3278 & 1.9617 & 4.3623 \\
\hline 1500 & 4.1383 & 6.1956 & 13.9487 \\
\hline
\end{tabular}




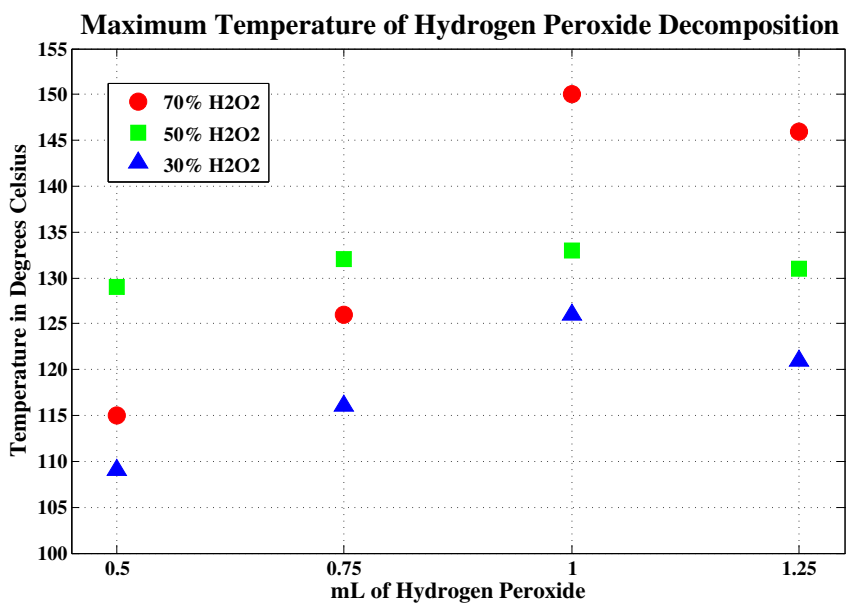

Fig. 7. The maximum temperature recorded directly underneath the catalyst screen during the decomposition reaction.

visible (where there is little visualization benefit to further inflation), does not require more initial fluid than a capsule's volume for $50 \%$ and $70 \% \mathrm{H}_{2} \mathrm{O}_{2}$.

These experiments support the feasibility of carrying a sufficient volume of fluid onboard a capsule to enhance visualization in the large intestine. Such a system could conceptually work in at least three different ways. First, a fluid reservoir could be added to an existing commercial camera pill and activated to enhance visualization when necessary. Second, a purely insufflation capsule could be built that does not contain a camera and swallowed in close succession with a regular commercial camera pill. Since passage through the colon takes up to 10 hours, both capsules would be in it at the same time. Activating the insufflation capsule would then inflate the entire colon, enhancing visualization for the commercial camera pill. Third, either of the first two strategies could be used with other types of capsules (e.g. magnetically guided capsules and those with other means of locomotion, see [2] for an overview) to ease their passage through the intestine and aid in locomotion.

We also note that a couple of safety issues would have to be addressed in such a future insufflation capsule. First, the amount of insufflation produced by the capsule should achieve optimal distention with minimal patient discomfort. In comparison to traditional colonoscopy, which can use a total of 8L of air [8], the amount of insufflation required here is much smaller. Further, the capsule would have to ensure that no liquid $\mathrm{H}_{2} \mathrm{O}_{2}$ was spilled on the intestine wall, as higher concentrations can cause some tissue damage if directly applied. Additionally, $50 \%-70 \% \mathrm{H}_{2} \mathrm{O}_{2}$ reacts at temperatures ranging from $115-150^{\circ} \mathrm{C}$ depending on the initial fluid amount and the concentration, as shown in Figure 7. To address this, we foresee using an insulated capsule shell that would dissipate the heat generated slowly as the capsule moves along the intestine and provide a buffer zone between the catalyst screen and the wall of the GI Tract. We note that the temperatures measured in our experiment may be higher than the actual temperature of the gas released, since they were recorded at the bottom of the flask directly underneath the reaction. However, careful experimentation will be needed in the future to ensure that thermal effects do not reach dangerous levels to tissue. It may even be possible to turn this "problem" into an advantage in the future, harnessing exothermal effects to intentionally deliver thermal therapy. One could envision a fluid-powered capsule specifically designed for ablation of cancerous tissues and/or cautery.

\section{CONCLUSION}

Our feasibility studies suggest that it is possible for a capsule to carry a sufficient volume of onboard fluid to provide a significant benefit to visualization in the GI Tract via insufflation. The results of our inflation experiment, in which we used a length of porcine large intestine replicating the geometry and volume of the average adult human colon, indicate that even small amounts of insufflation substantially increase the surface area of the intestine that is visualizable by the capsule.

In future work, we intend to build on the initial feasibility studies in this paper and design a prototype insufflation capsule. Initial versions will be built from off-the-shelf components as was done in [7], but with optimized internal capsule layout and enhanced materials. We can also foresee later versions that utilized customized micro-valves specifically designed for the application, which would likely have a smaller form factor and less stringent electrical requirements, thereby enabling more fluid to be carried on the capsule. Integrating wireless control electronics on such a capsule will also be essential. While there are clearly a number of technical challenges left to overcome, and safety will have to be considered in design and rigorously assessed experimentally, we believe that in this paper we have described the first steps toward an exciting new capability for wireless capsules in the GI Tract - the ability to insufflate tetherlessly. We believe that this has the potential to significantly increase the information content in capsule images, thus enhance reducing the incidence of false negative diagnoses.

\section{REFERENCES}

[1] A. Moglia, A. Menciassi, P. Dario, and A. Cuschieri, "Capsule endoscopy: progress update and challenges ahead," Nat. Rev. Gastroenterol. Hepatol., vol. 6, pp. 353-362, 2009.

[2] J. L. Toennies, G. Tortora, M. Simi, P. Valdastri, and R. J. Webster III, "Swallowable medical devices for diagnosis and surgery: The state of the art," Journal of Mechanical Engineering Science, 2010 (In Press).

[3] K. Twomey and J. R. Marchesi, "Swallowable capsule technology: current perspectives and future directions," Endoscopy, vol. 41, pp. 357-362, 2009.

[4] A. Menciassi, M. Quirini, and P. Dario, "Microrobotics for future gastrointestinal endoscopy," Minimally Invasive Therapy and Allied Technologies, vol. 16, pp. 91-100, 2007.

[5] M. Quirini, A. Menciassi, S. Scapellato, P. Dario, F. Rieber, C.-N. Ho, S. Schostek, and M. Schurr, "Feasibility proof of a legged locomotion capsule for the GI tract," Gastrointestinal Endoscopy, vol. 67, pp. 1153 $1158,2008$.

[6] R. Walker, Guide to the Human Body, J. Potts, Ed. Firefly Books Ltd., 2004.

[7] J. L. Toennies and R. J. Webster III, "A wireless insufflation system for capsular endoscopes," ASME Journal of Medical Devices, vol. 3, no. 2, p. $27514,2009$.

[8] M. Bretthauer, G. Hoff, E. Thiis-Evensen, G. Huppertz-Hauss, and E. Skovlund, "Air and carbon dioxide volumes insufflated during colonoscopy," Gastrointestinal Endoscopy, vol. 58, pp. 203-206, 2003. 